\title{
Double-configuration grating monochromator for extreme-ultraviolet ultrafast pulses
}

\author{
L. Poletto, ${ }^{1, \star}$ P. Miotti, ${ }^{1}$ F. Frassetto, ${ }^{1}$ C. Spezzani, ${ }^{2}$ C. Grazioli, ${ }^{2,3}$ \\ M. Coreno, ${ }^{2,4}$ B. Ressel, ${ }^{3}$ D. Gauthier, ${ }^{3}$ R. Ivanov,${ }^{5}$ A. Ciavardini, ${ }^{4}$ \\ M. de Simone, ${ }^{6}$ S. Stagira, ${ }^{7}$ and G. De Ninno ${ }^{2,4}$ \\ ${ }^{1}$ CNR - Institute of Photonics and Nanotechnologies, via Trasea 7, 35131 Padova, Italy \\ ${ }^{2}$ Elettra-Sincrotrone Trieste, S.S. 14-km 163,5 AREA Science Park, 34149 Trieste, Italy \\ ${ }^{3}$ University of Nova Gorica—Laboratory of Quantum Optics, Vipavska 11c, SI-5270 Ajdovscina, Slovenia \\ ${ }^{4}$ CNR_ISM, via Salaria km 29,300-00016 Monterotondo Scalo (RM), Italy \\ ${ }^{5}$ DESY, Notkestrasse, 85, Hamburg, 22607, Germany \\ ${ }^{6}$ CNR_IOM, S.S. 14-km 163,5 AREA Science Park, 34149 Trieste, Italy \\ ${ }^{7}$ Politecnico of Milano-Physics Department, P.zza L. Da Vinci 32, 20133 Milano, Italy \\ *Corresponding author: poletto@dei.unipd.it
}

Received 9 June 2014; revised 30 July 2014; accepted 31 July 2014; posted 4 August 2014 (Doc. ID 213657); published 4 September 2014

\begin{abstract}
We present the design and characterization of a double-configuration grating monochromator for the spectral selection of extreme-ultraviolet ultrafast pulses. Two grating geometries are joined in an instrument with two interchangeable diffracting stages, both used at grazing incidence: one with the gratings in the off-plane mount (OPM), the other in the classical diffraction mount (CDM). The use of two stages gives great flexibility: the OPM stage is used for sub-50 fs time response and low spectral resolution, while the CDM stage is for 100-200 fs time response and high spectral resolution. The monochromator spectral and temporal performances have been experimentally demonstrated on a high-order laserharmonics beam line.

OCIS codes: $\quad$ (050.1960) Diffraction theory; (320.0320) Ultrafast optics; (320.7160) Ultrafast technology; (340.7480) X-rays, soft x-rays, extreme ultraviolet (EUV).
\end{abstract}

\section{Introduction}

High-order laser harmonics (HHs), which are generated through nonlinear interaction between atoms and ultrafast laser pulses, are widely used to realize coherent, brilliant, ultrafast tabletop sources in the extreme ultraviolet (XUV) and soft x-ray regions [1]. The $\mathrm{HH}$ spectrum is described as a sequence of peaks corresponding to odd harmonics of the fundamental laser wavelength with an intensity distribution characterized by a plateau whose extension is related to the pulse intensity and laser wavelength. The combination of the use of advanced phase matching mechanisms and interaction geometries has made it possible to obtain $\mathrm{HH}$ radiation up to the water window region $(2.3-4.4 \mathrm{~nm})[2,3]$. Moreover, the radiation generated with the scheme of the HHs using few optical cycle laser pulses is nowadays the main tool for investigating matter with sub-femtosecond temporal resolution [4-6].

Spectroscopic applications of $\mathrm{HHs}$ often require the spectral selection of a single harmonic in a narrow XUV band, especially for experiments aimed to 
gain insight into electronic structures of materials, where multiple orders of $\mathrm{HHs}$ have to be filtered out. Although multilayer mirrors are the simplest optical elements for the spectral selection of one harmonic order and are successfully employed for $\mathrm{HHs}$ and synchrotron beam lines, they may give a poor contrast ratio between adjacent harmonics [7].

A grating monochromator, although more complex than a single multilayer mirror, gives both tunability in a broad range of harmonics and high spectral selectivity. However, it introduces a stretch of the pulse duration because of the pulse-front tilt, compromising the ultrafast duration of $\mathrm{HHs}$, as schematically shown in Fig. 1. This effect, although almost negligible in the picosecond or longer time scale, may completely alter the temporal duration of the XUV pulse in the femtosecond regime. Nevertheless, it is possible to design grating monochromators that do not alter the temporal duration of ultrafast pulses by using two gratings in a time-delay compensated configuration, where the second grating compensates for the front tilt and for the spectral spread introduced by the first one [8-12]. Pulses as short as 8-10 fs have been measured at the output of double-grating monochromators in the $20-45 \mathrm{~nm}$ spectral region [13-16]. The main drawback of these configurations is the use of two gratings that increase the complexity of the instrument and reduce the efficiency.

Let us analyze the condition for minimum temporal broadening from a single grating used at the first diffraction order. The minimum number of illuminated grooves $N_{\min }$ to support a bandwidth $\Delta \lambda$ at wavelength $\lambda$ can be found using the relation $N_{\min }=$ $\lambda / \Delta \lambda$. Since the absolute delay given by each adjacent groove is $\lambda$, the front tilt given by $N$ illuminated grooves is $\lambda N$. The corresponding minimum pulse front tilt at half-width, $\Delta \tau_{G \text {,min }}$, for a given bandwidth $\Delta \lambda$ is

$$
\Delta \tau_{G, \min } \cong \frac{1}{2} \frac{\lambda N_{\min }}{c}=0.5 \frac{\lambda^{2}}{c \Delta \lambda} .
$$

$\Delta \tau_{G, \min }$ is close to $\Delta \tau$, the Fourier limit of a Gaussian pulse with no phase modulation:

$$
\Delta \tau=\frac{2 \ln 2}{\pi} \frac{\lambda^{2}}{c \Delta \lambda} \cong 0.44 \frac{\lambda^{2}}{c \Delta \lambda} .
$$

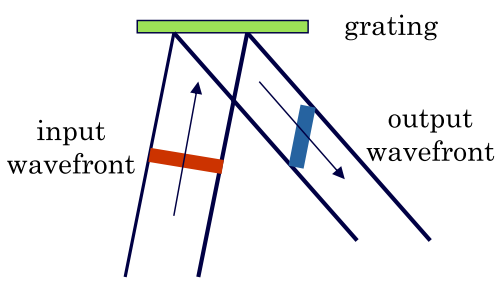

Fig. 1. Pulse front-tilt given by a diffraction grating. The tilt of the output wavefront is $N m \lambda$, where $N$ is the number of illuminated grooves, $m$ is the diffraction order, $\lambda$ is the wavelength.
If a transform-limited pulse passes through a monochromator with minimum pulse front tilt, the minimum pulse duration is the convolution of Eqs. (1) and (2). Therefore, a single grating may be used for spectral selection of ultrafast pulses at the first diffraction order without altering in a significant way the pulse duration, provided that the number of illuminated grooves is equal to the actual resolution.

The optical design of monochromators for ultrafast pulses aims, thus, at optimizing the configuration, which gets closest to this condition.

Two different grating configurations can be adopted for grazing-incidence diffraction [17]: the classical diffraction mount (CDM) and the off-plane mount (OPM). We discuss the advantages and drawbacks of both mountings for application to the spectral selection of ultrafast pulses and present a configuration where the two geometries are joined in a single instrument, here called the doubleconfiguration monochromator (DCM), which overcomes the limits of each of the two configurations. The design allows realizing a broadband grazingincidence single-grating monochromator with either ultrafast time response with low spectral resolution or longer time response with higher resolution.

A DCM for ultrafast $\mathrm{HHs}$ has been recently realized in the framework of the CITIUS project. The aim of the project is to set up a new state-of-the-art ultrafast light source based on $\mathrm{HH}$ generation at Nova Gorica University (Slovenia). The CITIUS facility [18] is based on a high repetition rate and ultrashort tunable laser source, which is used to produce ultrafast XUV pulses through $\mathrm{HH}$ generation. The laser system is a $3 \mathrm{~mJ}, 40 \mathrm{fs}, 5 \mathrm{kHz}$ Ti:Sapphire operated at $805 \mathrm{~nm}$ (Legend Elite Duo, Coherent, USA). One third of the output energy, i.e., $1 \mathrm{~mJ}$, is split and used to drive a widely tunable OPA system, which provides pulses from $240 \mathrm{~nm}$ to $2.6 \mu \mathrm{m}$ that can be used for pump-probe experiments. The remaining energy, i.e., $2 \mathrm{~mJ}$, is used to generate HHs. The vacuum beam line is schematically shown in Fig. 2 . It includes (a) the $\mathrm{HH}$ generation chamber; (b) and (c) the monochromator chamber and the exit slit block; (d) the calibrated photodiode that can be inserted in the beam path in order to measure the XUV photon flux; (e) a metallic filter that can be possibly inserted in the XUV path to cut the diffused IR light; (f) the XUV refocusing section, which hosts a

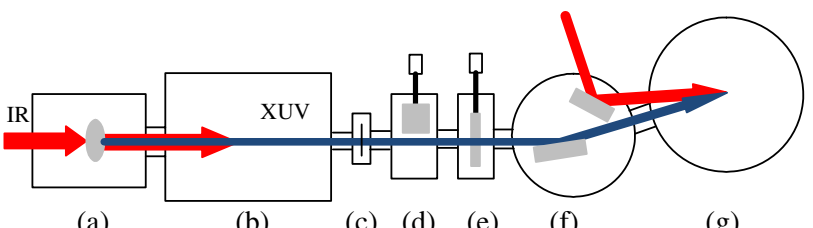

(a)

(b)

(c) (d) (e)

(f)

(g)

Fig. 2. Schematic of the XUV photon handling section of the CITIUS facility. (a) HH generation chamber with gas cell. (b) Monochromator chamber. (c) Exit slit. (d) Insertable calibrated photodiode. (e) Insertable metallic filter. (f) XUV-IR recombination and refocusing chamber. (g) Experimental chamber. 
grazing-incidence toroidal mirror to focus the XUV monochromatized beam; and a plane mirror used to recombine the IR for pump-probe experiments on (g) the experimental chamber. The XUV and IR beams are recombined almost collinearly, with an angle of about $2^{\circ}$.

The CITIUS facility aims to combine femtosecond optical science and synchrotron technologies to enable ultrafast pump-probe experiments. The scientific program will be carried out in close connection with the low-density matter (LDM) beamline at the FERMI@Elettra free-electron laser, in a wide range of applied and fundamental fields, including material science, catalysis, biochemistry, and magnetism.

In this paper, we discuss the design of the monochromator and present its characterization in terms of absolute photon output, spectral resolution, imaging properties, and temporal response.

\section{Grating Configurations for Ultrafast Monochromators}

The CDM is shown in Fig. 3(a), and the layout of a plane-grating monochromator is shown in Fig. 4(a). The design consists of three optical elements: two toroidal mirrors and the plane grating with uniform line spacing that is operated in collimated light. The first mirror acts as the collimator and the second one as the condenser, which focuses the beam on the exit slit. The mirrors are operated at equal angle and unity magnification to minimize aberrations at the output. The wavelength selection is performed by rotating the grating around the axis, which is tangent to the surface, passes through the grating center, and is parallel to the grooves, following the relation:

$$
\alpha=\frac{K}{2}+\arcsin \left[\frac{m \lambda \sigma_{c}}{2 \cos (K / 2)}\right], \quad \beta=K-\alpha,
$$

where $\alpha$ and $\beta$ are, respectively, the incidence and diffraction angles (both defined with positive signs), $K=\alpha,+\beta$ is the subtended angle, and $\sigma_{c}$ the groove density. The number of illuminated grooves is $N_{c}=S \sigma_{c} / \cos \alpha$, where $S$ is the section of the collimated beam. The illuminated grooves are lower in the case of $m=-1$, i.e., the external diffracted order, since $\alpha$ is smaller. The exit slit has to be placed vertically.

The OPM is shown in Fig. 3(b), and the layout of a plane-grating monochromator is shown in Fig. 4(b). As for the CDM, the design consists of the plane grating with uniform line spacing and two toroidal

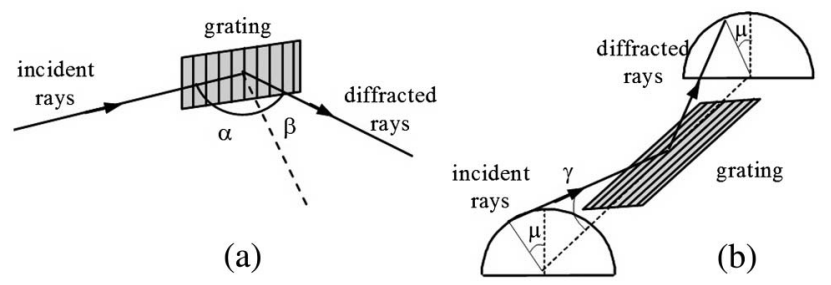

Fig. 3. (a) CDM grating. (b) OPM grating.

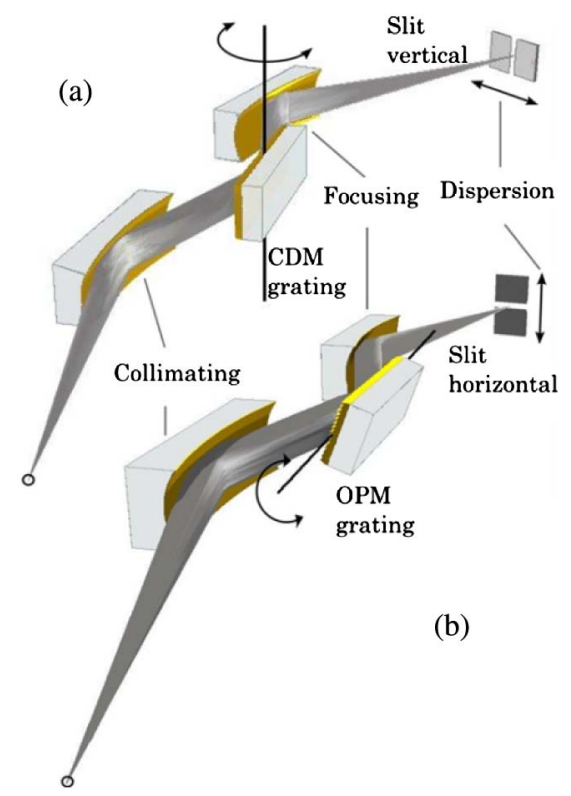

Fig. 4. Layout of a plane-grating monochromator in (a) CDM and (b) OPM geometry.

mirrors. The wavelength selection is performed by rotating the grating around the axis, which is tangent to the surface, passes through the grating center, and is parallel to the grooves, following the relation

$$
\mu=\arcsin \left[\frac{m \lambda \sigma_{o}}{2 \sin \gamma}\right]
$$

where $\sigma_{o}$ is the groove density, $\gamma$ is the altitude angle, and $\mu$ is the azimuth of the incoming and diffracted rays. The number of illuminated grooves is $N_{o}=$ $S \sigma_{o} / \cos \mu \approx S \sigma_{o}$, where the approximation holds for small $\mu\left(\mu \leq 15^{\circ}\right)$ and is fully verified for lowresolution configurations. The grating in the OPM can be used indifferently at the orders $m= \pm 1$, since this does not affect the illuminated area. The exit slit has to be placed horizontally.

Once the exit arm of the focusing mirror, $p$, has been defined, the output bandwidth is given by

$$
\begin{aligned}
& \Delta \lambda_{c}=\frac{\cos \beta}{m \sigma_{c} p} \Delta s, \\
& \Delta \lambda_{o}=\frac{1}{m \sigma_{o} p} \Delta s,
\end{aligned}
$$

where $\Delta s$ is the width of the exit slit. Equation ( $\underline{5})$ refers to the CDM; Eq. ( $\underline{6})$ refers to the OPM.

In both configurations, the diffraction efficiency is maximized in the blaze condition, which is verified when the diffracted light leaves the grating in such a way to perform a specular reflection on the groove surface. In case of saw-tooth profiles with blaze angle $\delta$, the condition is fulfilled for $\delta=(\alpha-\beta) / 2$ in the CDM and for $\delta=\mu$ in the OPM. 
The main advantage of the OPM when compared with the CDM is the higher diffraction efficiency, which has been measured to be actually close to the coating reflectivity at the same altitude [19-22]. On the other hand, the main drawback is the distortion and the rotation of the image after the diffraction, which is due to the highly nonspecular reflection from the grating, especially for the large azimuth angles, which are required to achieve high spectral resolution [23]. This makes the OPM much more complex to be adopted than the CDM for highresolution designs. Indeed, for low-resolution designs, as those required for ultrafast pulses, the distortion and rotation are almost negligible, since the azimuth angle is low.

The two geometries here are compared in their application to ultrafast monochromators, outlining respective advantages and drawbacks. Here and in the following, it is assumed that the grating is operated in the first diffracted order, as is typical in most cases. The half-width beam section on the grating is assumed to be $1 \mathrm{~mm}$. We analyze two different temporal regimes: 100 or 20 fs half-width pulse front tilt at $30 \mathrm{~nm}(41 \mathrm{eV})$. The corresponding maximum number of illuminated grooves to support such a time response is 2000 for $100 \mathrm{fs}$ and 400 for $20 \mathrm{fs}$. The grating groove density (as a function of the subtended angle and of the altitude for, respectively, CDM and OPM) calculated to support the front tilt mentioned above is reported in Table 1 . In the case of the CDM, as the subtended angle increases, the corresponding groove density decreases. In the case of the OPM, the groove density is almost independent from the choice of the altitude angle.

The blaze angle that maximizes the grating efficiency in the CDM is definitely lower than that in the OPM. Although the theoretical diffraction efficiency is expected to be close to the coating reflectivity at the same angle in both configurations, the measured efficiency is critically dependent on the actual groove profile, particularly in the CDM, since the unavoidable smoothing of the sharp edges in the real cases contributes to a rather strong decrease of efficiency with respect to the theoretical values $[24,25]$. This effect is more evident for extreme blaze angles, where the realization of an ideal saw-tooth profile is practically unfeasible.

CDM monochromators with front tilts in the range of hundreds of femtoseconds require the use of gratings with blaze angles around $1^{\circ}$, which are well within the present capabilities of manufacturers. Instead, front tilts in the range of a few tens of femtoseconds require an extremely low groove density in the CDM and a blaze angle that is practically unfeasible, so that laminar profiles have to be adopted with extremely low efficiency. In contrast, the blaze angles required in the OPM configurations are less extreme than in the CDM. This gives a lower deviation between the ideal and actual saw-tooth profile and contributes to the increase of efficiency with respect to the CDM.
Table 1. Grating Parameters to Support 20 or 100 fs Half-Width Pulse Front Tilt at $30 \mathrm{~nm}^{\mathrm{a}}$

\begin{tabular}{|c|c|c|c|}
\hline \multicolumn{4}{|c|}{$20 \mathrm{fs}$ Pulse Front Tilt at $30 \mathrm{~nm}$} \\
\hline \multicolumn{4}{|c|}{ Classical Diffraction Mount } \\
\hline $\begin{array}{l}\text { Subtended } \\
\text { Angle }\left(^{\circ}\right)\end{array}$ & $\begin{array}{l}\text { Groove Density } \\
\text { (gr/mm) }\end{array}$ & $\begin{array}{c}\text { Incidence } \\
\text { at } 30 \mathrm{~nm}\left({ }^{\circ}\right)\end{array}$ & $\begin{array}{c}\text { Blaze } \\
\text { Angle }\left(^{\circ}\right)\end{array}$ \\
\hline 155 & 44 & 77.3 & 0.2 \\
\hline 160 & 36 & 79.8 & 0.2 \\
\hline 165 & 27 & 82.3 & 0.2 \\
\hline \multicolumn{4}{|c|}{ Off-Plane Mount } \\
\hline Altitude $\left(^{\circ}\right)$ & $\begin{array}{l}\text { Groove Density } \\
\text { (gr/mm) }\end{array}$ & $\begin{array}{c}\text { Azimuth } \\
\text { at } 30 \mathrm{~nm}\left(^{\circ}\right)\end{array}$ & $\begin{array}{c}\text { Blaze } \\
\text { Angle }\left(^{\circ}\right)\end{array}$ \\
\hline 5 & 200 & 2.0 & 2.0 \\
\hline 7 & 200 & 1.4 & 1.4 \\
\hline 10 & 200 & 1.0 & 1.0 \\
\hline \multicolumn{4}{|c|}{$100 \mathrm{fs}$ Pulse Front Tilt at $30 \mathrm{~nm}$} \\
\hline \multicolumn{4}{|c|}{ Classical Diffraction Mount } \\
\hline $\begin{array}{l}\text { Subtended } \\
\text { Angle }\left(^{\circ}\right)\end{array}$ & $\begin{array}{l}\text { Groove Density } \\
\text { (gr/mm) }\end{array}$ & $\begin{array}{c}\text { Incidence } \\
\text { at } 30 \mathrm{~nm}\left({ }^{\circ}\right)\end{array}$ & $\begin{array}{c}\text { Blaze } \\
\text { Angle }\left(^{\circ}\right)\end{array}$ \\
\hline 155 & 233 & 78.4 & 0.9 \\
\hline 160 & 190 & 79.1 & 0.9 \\
\hline 165 & 148 & 81.5 & 1.0 \\
\hline \multicolumn{4}{|c|}{ Off-Plane Mount } \\
\hline Altitude $\left(^{\circ}\right)$ & $\begin{array}{c}\text { Groove Density } \\
(\mathrm{gr} / \mathrm{mm})\end{array}$ & $\begin{array}{c}\text { Azimuth } \\
\text { at } 30 \mathrm{~nm}\left({ }^{\circ}\right)\end{array}$ & $\begin{array}{c}\text { Blaze } \\
\text { Angle }\left({ }^{\circ}\right) \\
\end{array}$ \\
\hline 5 & 990 & 9.8 & 9.8 \\
\hline 7 & 1000 & 7.1 & 7.1 \\
\hline 10 & 1000 & 5.0 & 5.0 \\
\hline
\end{tabular}

${ }^{a}$ Half-width beam section on the grating is assumed to be $1 \mathrm{~mm}$.

The calculations presented above can be generalized as summarized in Fig. 5. The CDM and OPM are compared at two wavelengths in terms of grating characteristics for front tilts in the 10-200 fs range. For front tilts below $100 \mathrm{fs}$, the blaze angles required for gratings in the CDM are beyond the present capabilities of manufacturers, while the CDM requires groove densities and blaze angles that are feasible and give high efficiency. For front tilts longer than $100 \mathrm{fs}$, the OPM requires high azimuth angles especially for short wavelengths; this is not a suitable configuration, since it introduces a large anamorphic deformation.

As a general claim, the CDM should be preferred for monochromators when relatively large front tilts, i.e., 100-200 fs, are accepted and medium-high spectral resolution is required, while the OPM has to be adopted for ultrafast responses in the $10-50$ fs range and low spectral resolution.

\section{Double-Configuration Ultrafast Monochromator}

The two grating geometries discussed above can be joined in a design of a DCM with either ultrafast time response with low spectral resolution from the OPM or longer time response with higher resolution from 

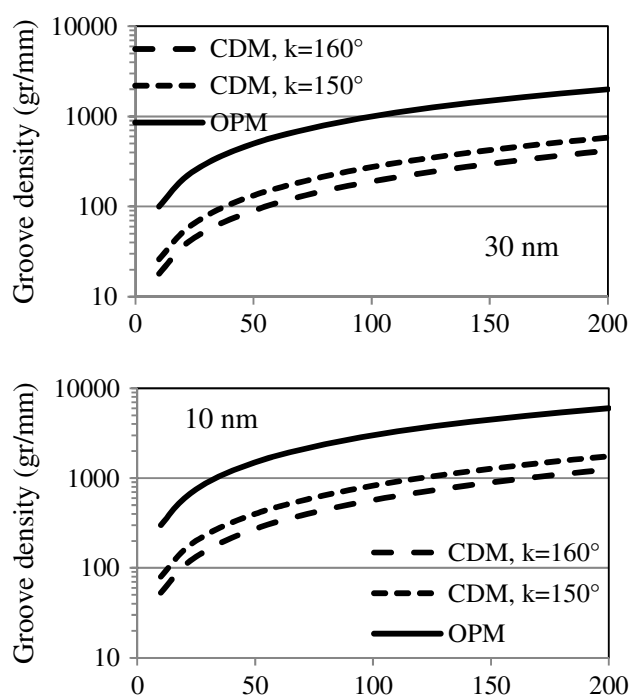

(a)

(b)

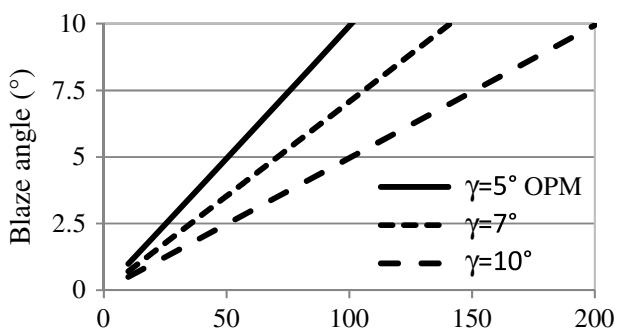

(c)

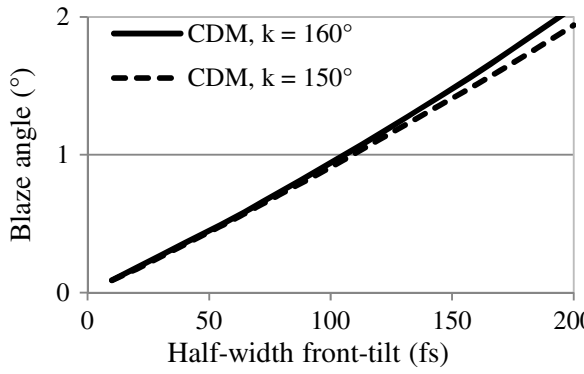

(d)

Fig. 5. Ultrafast monochromator in CDM and OPM for $1 \mathrm{~mm}$ half-width beam section on the grating. (a) and (b) Groove density as a function of the pulse front tilt at (a) $30 \mathrm{~nm}$ and (b) $10 \mathrm{~nm}$. (c) Corresponding blaze angle for the OPM at three altitudes. (d) Corresponding blaze angle for the CDM at two subtended angles.

the CDM. Although CDM monochromators are the standard elements for the spectral selection of $\mathrm{HHs}$ and have been used since the pioneering work in this field [26], and although the OPM has been already applied to the realization of single-stage ultrafast monochromators [27], to the authors' knowledge, this is the first time that both geometries are joined together in a single instrument.

The DCM has two different and interchangeable grating stages: the former with the gratings in the OPM and the latter in the CDM. The use of two stages gives great flexibility: the OPM stage is used for sub-50 fs time response and low spectral resolving power $(\lambda / \Delta \lambda<100)$, and the CDM stage is used for 100-500 fs time response and medium resolving power $(\lambda / \Delta \lambda>500)$. In this way, any user can choose
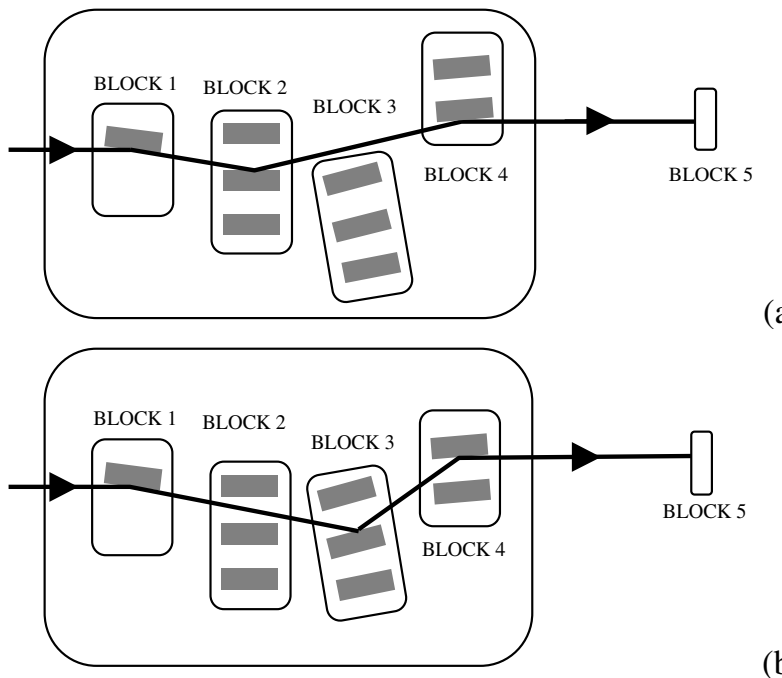

(a)

Fig. 6. DCM schematic view. (a) OPM configuration: light is travelling through the OPM grating and related focusing mirror. (b) CDM configuration: light is travelling through the CDM grating and the related focusing mirror. Block 1 is collimating toroidal mirror; Block 2 the OPM gratings stage; Block 3 the CDM gratings stage; Block 4 the focusing toroidal mirrors. The exit slit block is placed $400 \mathrm{~mm}$ away from the focusing mirror and is not indicated in the drawing.

the most suitable monochromator configuration for the experiment by insertion of the proper stage in the optical path.

A schematic drawing of the DCM is shown in Fig. 6 . Block 1 is the toroidal mirror, which collimates light coming from the $\mathrm{HH}$ source. Block 2 consists of three OPM gratings (manufactured by NewportRichardson Grating), operated at the same altitude angle, which are mounted on two motorized stages: (1) rotating stage for the wavelength scanning (changing the azimuth angle) and (2) linear one for the grating selection. Block 2 can be automatically removed from the optical path to let the beam propagate to the following CDM gratings (also manufactured by Newport-Richardson Grating), which are mounted on Block 3 and operated at a constant deviation angle. Similarly to Block 2, Block 3 has a rotating stage for the wavelength scanning (changing the incidence angle) and a linear one for the grating selection. Block 4 has two toroidal mirrors that can be inserted in the optical path to focus the light on the slit: the first one is demanded to intercept the light diffracted by the grating in the OPM; the other is used with the CDM. Finally, Block 5 accommodates two variable-width slits mounted in close proximity: the former is horizontal for the OPM, the latter vertical for the CDM. An internal view of the monochromator chamber is shown in Fig. 7. The DCM parameters are summarized in Table $\underline{2}$. The monochromator bandwidth is shown in Fig. 8 .

Based on measurements of single-stage configurations using similar gratings and reported in $[14,25,27]$, the throughput of the monochromator is estimated in the range $0.15-0.20$ for the CDM stage and $0.35-0.40$ for the OPM stage. 


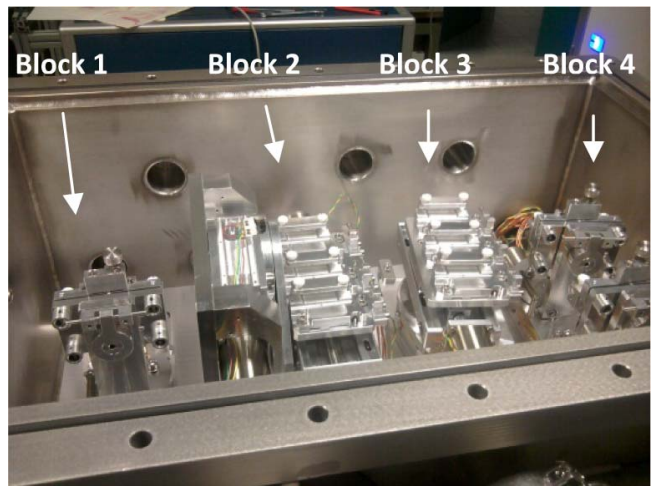

Fig. 7. Internal view of the DCM chamber. The size of the chamber is $650 \mathrm{~mm} \times$ (length) $\times 350 \mathrm{~mm} \times$ (width).

\section{Monochromator Characterization}

XUV radiation in the wavelength range 15.5$88.5 \mathrm{~nm}(80-14 \mathrm{eV})$ is produced by $\mathrm{HH}$ generation in a gas cell, using $40 \mathrm{fs}, 2 \mathrm{~mJ}$ Ti:Sa laser pulses at $5 \mathrm{kHz}$ repetition rate. The IR beam is focused in the gas cell by a lens. The IR co-propagates with the HHs up to the grating (Block 3 in OPM, Block 4 in CDM). When the grating is rotated to perform the XUV wavelength scanning, the IR beam is totally diffracted on the 0th order out of the optical axis and is blocked by a suitable beam stop placed before the output mirror (Block 4).

The absolute monochromatic XUV photon flux is measured after the exit slit with a calibrated $\mathrm{Al}$ photodiode (provided by NIST, USA), which can be inserted in the optical path.

The beam passes, then, possibly through a $200 \mathrm{~nm}$ thick Al filter, which may be inserted in the optical path to stop the residual diffused IR light, and enters the recombination chamber, where a grazingincidence toroidal mirror (Au coated, $1400 \mathrm{~mm}$ focal length) focuses the monochromatized XUV light at the sample position with unity magnification. The toroidal mirror sits on two motorized mounts to control the mirror rotations that adjust the $x-y$ position of the output spot. The spatial overlap of the two XUV foci when the two different paths are selected, i.e., OPM either CDM grating stages, is guaranteed with micrometric precision. The IR beam for pumpprobe experiments is recombined with the XUV in an almost collinear geometry through a plane mirror, which is remotely controlled to perform an almost perfect spatial overlap between the pump and probe beams onto the sample. The arms of the refocusing mirror have been selected to be rather long, i.e., $1400 \mathrm{~mm}$, in order to accommodate also large experimental chambers.

\section{A. Spectral Response}

Some typical scanned HH spectra are shown in Fig. $\underline{9}$. The use of Ar as the interacting gas gives harmonics in the photon energy range of $20-54 \mathrm{eV}$, with a peak emission in the H19-H25 interval (29-39 eV) and a cutoff at $51 \mathrm{eV}(\mathrm{H} 33)$. Using $\mathrm{Ne}$, the plateau region

Table 2. Parameters of $\mathrm{DCM}^{\mathrm{a}}$

\begin{tabular}{|c|c|c|c|}
\hline \multicolumn{2}{|l|}{ MIRRORS } & \multicolumn{2}{|l|}{ GRATINGS } \\
\hline Source-to-mirror distance & $400 \mathrm{~mm}$ & Altitude & $5^{\circ}$ \\
\hline \multirow[t]{2}{*}{ Radii } & $15300 \mathrm{~mm} \times 41.9 \mathrm{~mm}$ & Groove density & $200 \mathrm{gr} / \mathrm{mm}$ \\
\hline & & Blaze angle & $4.2^{\circ}$ \\
\hline BLOCK 4 & Focusing toroidal mirrors & Blaze wavelength & $63 \mathrm{~nm}(19.7 \mathrm{eV})$ \\
\hline Incident angle & $87^{\circ}$ & Blaze angle & $4.5^{\circ}$ \\
\hline Radii & $15300 \mathrm{~mm} \times 41.9 \mathrm{~mm}$ & Blaze wavelength & $32 \mathrm{~nm}(38.8 \mathrm{eV})$ \\
\hline Mirror 2 & Classical geometry & Grating 3 & $12-30 \mathrm{~nm}(100-40 \mathrm{eV})$ \\
\hline Incident angle & $80^{\circ}$ & Groove density & $600 \mathrm{gr} / \mathrm{mm}$ \\
\hline \multirow[t]{2}{*}{ Radii } & $4600 \mathrm{~mm} \times 139 \mathrm{~mm}$ & Blaze angle & $3.5^{\circ}$ \\
\hline & & Blaze wavelength & $18 \mathrm{~nm}(68.9 \mathrm{eV})$ \\
\hline Off-plane slit & Horizontal & Blaze angle & $4.3^{\circ}$ \\
\hline Classical-mount slit & Vertical & Blaze wavelength & $100 \mathrm{~nm}(12.4 \mathrm{eV})$ \\
\hline \multirow[t]{8}{*}{ Distance between the two slits } & $5 \mathrm{~mm}$ & Grating 2 & $20-60 \mathrm{~nm}(60-20 \mathrm{eV})$ \\
\hline & & Groove density & $600 \mathrm{gr} / \mathrm{mm}$ \\
\hline & & Blaze angle & $5.2^{\circ}$ \\
\hline & & Blaze wavelength & $68 \mathrm{~nm}(18.2 \mathrm{eV})$ \\
\hline & & Grating 3 & $12-30 \mathrm{~nm}(100-40 \mathrm{eV})$ \\
\hline & & Groove density & $1200 \mathrm{gr} / \mathrm{mm}$ \\
\hline & & Blaze angle & $7^{\circ}$ \\
\hline & & Blaze wavelength & $46 \mathrm{~nm}(27.0 \mathrm{eV})$ \\
\hline
\end{tabular}

${ }^{a}$ All optical elements are gold-coated. 


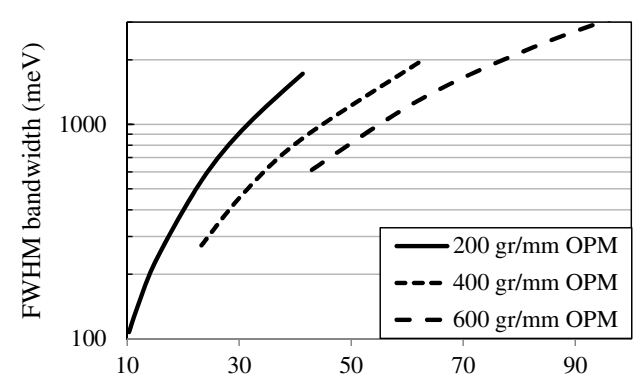

(a)

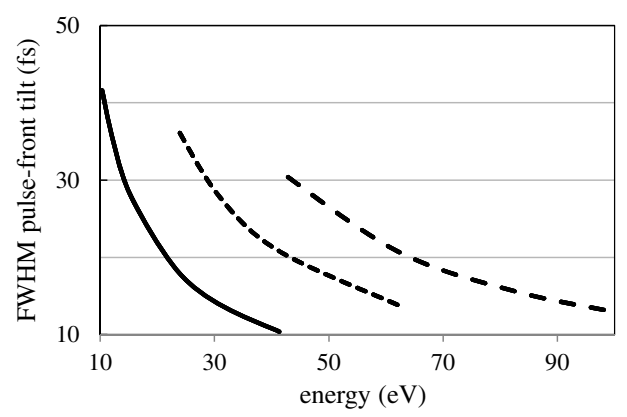

(c)

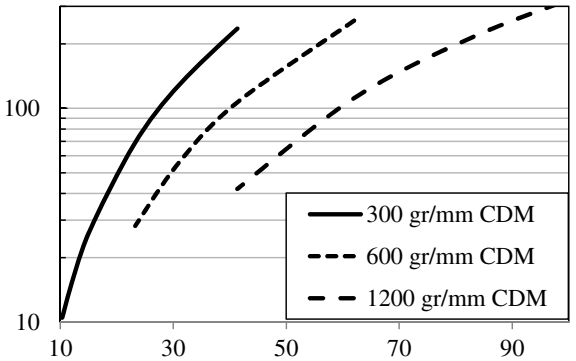

(b)

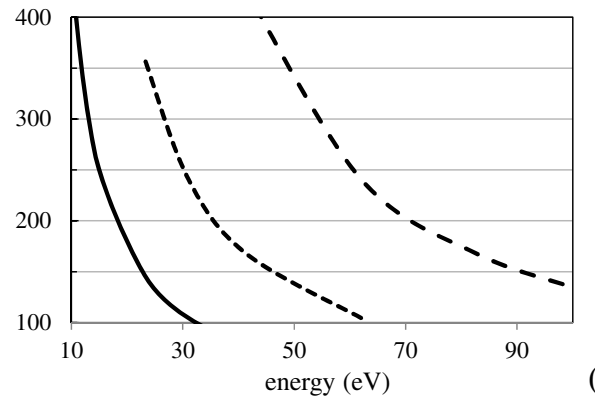

(d)

Fig. 8. Bandwidth on $100 \mu \mathrm{m}$ slit and pulse front tilt with $1.5 \mathrm{mrad}$ divergence: (a), (c) OPM; (b), (d) CDM.

and the cut-off harmonic move toward higher energies, which have been measured up to $79 \mathrm{eV}$ (H51) in the best operative conditions, although with significantly reduced efficiency. Note that the secondorder diffraction lines are clearly separated from the main harmonics.

\section{B. Photon Flux}

By using $2 \mathrm{~mJ} 5 \mathrm{kHz}$ laser pulses to generate HHs in argon, absolute photon flux as high as 5 . $10^{11}$ photons/s at $35.6 \mathrm{eV}$ (H23) has been measured at the output of the monochromator, corresponding to $10^{8}$ photons/pulse $=0.57 \mathrm{~nJ} /$ pulse on the whole harmonic bandwidth. Using $\mathrm{Ne}$, the flux is reduced by a factor $\approx 20$, which is $2.5 \cdot 10^{10}$ photons $/ \mathrm{s}, 5$. $10^{6}$ photons $/$ pulse $=28 \mathrm{pJ} /$ pulse at $35.6 \mathrm{eV}$ (H23). Maximum photon flux has been achieved with a $5 \mathrm{~mm}$ gas cell and a $76 \mathrm{~cm}$ focusing lens. The values have been measured with the OPM G400 grating and the exit slit open at $200 \mu \mathrm{m}$ to transmit the whole bandwidth of a single harmonic.

\section{Spot Size}

The size of the XUV spot after the refocusing mirror with a slit aperture of $200 \pm 10 \mu \mathrm{m}$ was measured at different harmonics by an XUV-enhanced CCD detector (PIXIS-XO 400B by Princeton Instruments) placed in the focal plane of the refocusing mirror. The measurements have been completed at a fixed aperture of the slit, $200 \mu \mathrm{m}$, for a wide range of harmonics, from $\mathrm{H} 11$ to $\mathrm{H} 23$, and with different gratings, both OPM and CDM. The spot size has been verified to be almost constant for all the gratings and the harmonics. It results in 120-140 $\mu \mathrm{m}$ ( $\|$ to the slit $) \times 220 \mu \mathrm{m} \times(\perp$ to the slit $)$. The $220 \mu \mathrm{m}$ transversal size of the image is in agreement with the slit aperture, since the refocusing mirror is working in $1: 1$ configuration. For example, the spot size of H21 of the CDM G1200 grating is shown in Fig. 10. A slight asymmetry is visible in the vertical cross section of Fig. 10(b), which indicates the presence of some aberration in the spot that is ascribed to the residual misalignment of the toroidal mirrors.

The effects of the imaging errors on the wavefront distortions have been simulated through ray tracing. Even in the case of a misalignment of the toroidal mirrors of $\pm 0.3^{\circ}$, which definitely exceeds the uncertainty in the alignment, the wavefront distortions are simulated below $5 \mathrm{fs}$. Therefore, it may be concluded that the main effect that influences the temporal response of the monochromator is the pulse front tilt given by the gratings.

\section{Beam Divergence}

The XUV beam divergence has been measured by a knife-edge test by taking the FWHM beam size at a known distance from the XUV source. In the case of a driving IR beam focused with a $76 \mathrm{~cm}$ lens in a $5 \mathrm{~mm}$ long gas cell, the measured FWHM divergence of the $\mathrm{HHs}$ generated in argon is $1.3 \mathrm{mrad}$ for $\mathrm{H} 15$ and $1.0 \mathrm{mrad}$ for H23, decreasing toward the cut-off harmonics [28].

\section{E. Pulse Duration}

The residual pulse front tilt at the final focus of the monochromatic beamline has been verified by the measurement of the temporal duration of the XUV pulse by the XUV-IR cross-correlation technique described in detail in [29] and [30].

The experiment has been performed using a commercial electron analyzer (Scienta SES-200 spectrometer). A gas jet ( $\mathrm{Kr}$, in the case here described) is injected in the focal point of the XUV beamline. The HH XUV pulse ionizes the target gas in the presence 

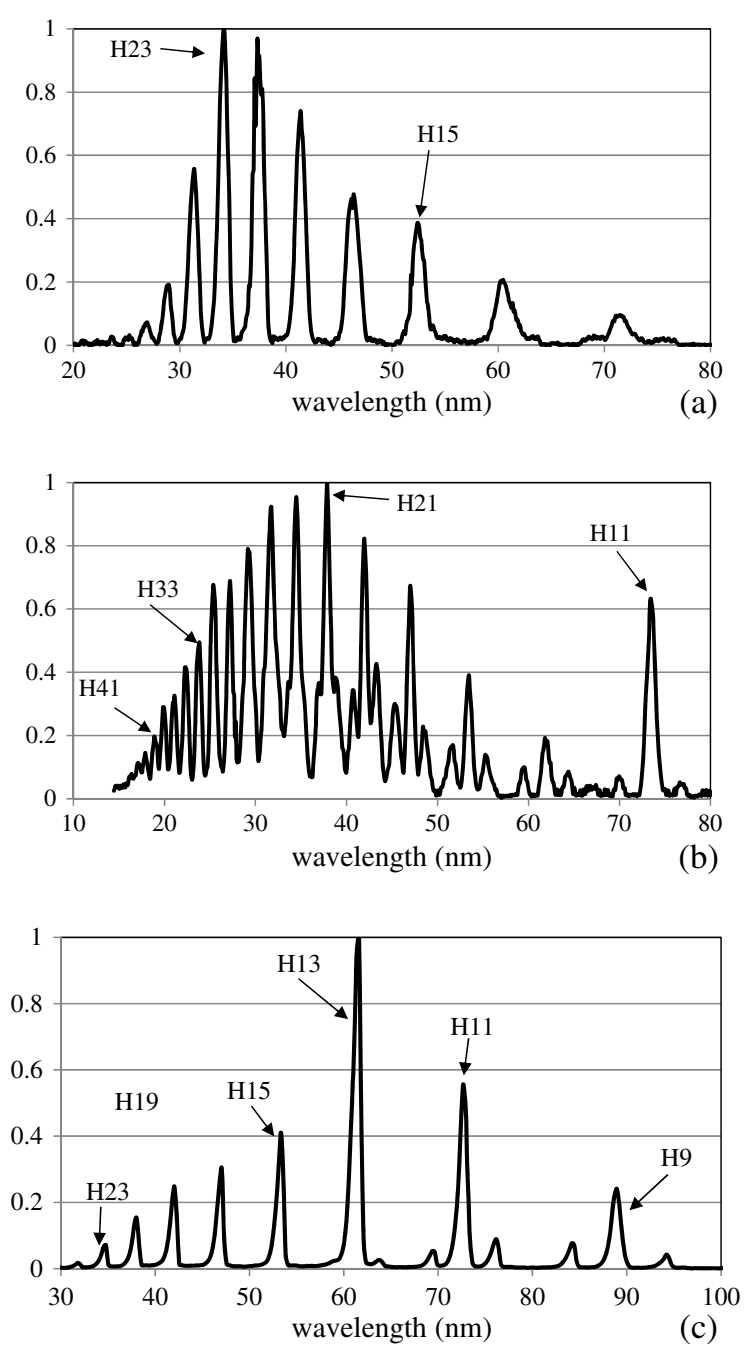

Fig. 9. HH spectra, $2 \mathrm{~mJ} 40 \mathrm{fs} 5 \mathrm{kHz}$ IR laser pulses, normalized intensity: (a) OPM G400 grating, argon, $76 \mathrm{~cm}$ focusing lens, $100 \mathrm{um}$ slit, $5 \mathrm{~mm}$ gas cell, generation parameters optimized to have the maximum emission at H23. (b) OPM G400 grating, neon, $76 \mathrm{~cm}$ focusing lens, $100 \mu \mathrm{m}$ slit, $10 \mathrm{~mm}$ gas cell, generation parameters optimized to have the maximum emission in the H19-H31 interval. (c) CDM G600 grating, argon, $40 \mathrm{~cm}$ focusing lens, $200 \mu \mathrm{m}$ slit, $5 \mathrm{~mm}$ gas cell, generation parameters optimized to have the maximum emission at H13.

of the IR field, which is spatially overlapped to the XUV. When the two pulses overlap also in time on the gas jet, sidebands appear in the photoelectron spectrum, spectrally shifted by the IR photon energy, determined by the absorption of one harmonic photon plus the absorption or the emission of one IR photon. The amplitude of the sidebands as a function of the delay between the XUV and IR pulses provides the cross-correlation signal from which one can extract the duration of XUV pulses. The IR pulse duration was measured to be $40 \pm 2 \mathrm{fs}$ after the IR transport beamline in the experimental chamber. The XUV-IR delay was controlled by a motorized piezoelectric stage translator with a resolution of $10 \mathrm{~nm}$. The experiment has been performed with the monochromator tuned at H15, i.e., $23.3 \mathrm{eV}$. The XUV photon has sufficient energy to ionize the $\mathrm{Kr} 4 p$

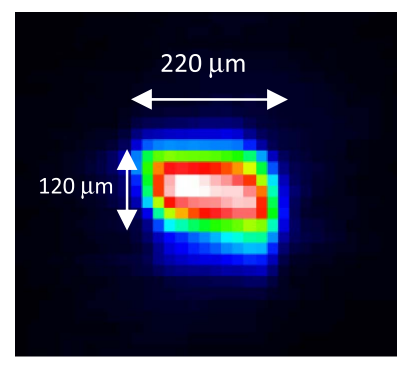

(a)

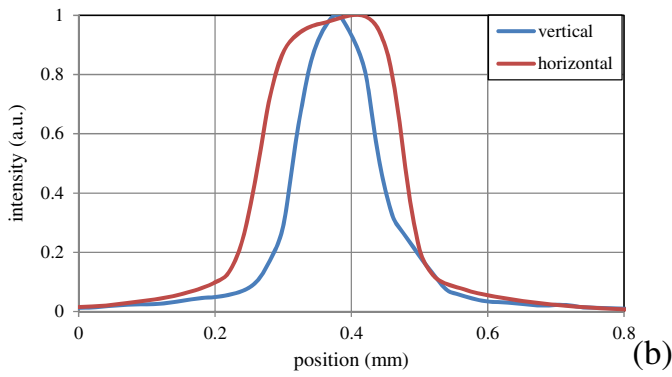

Fig. 10. XUV spot of $\mathrm{H} 21$ after the recombination mirror, with a slit aperture of $200 \pm 20 \mu \mathrm{m}, \mathrm{CDM}$ G1200 grating, imaged on a XUV-enhanced CCD camera ( $20 \mu \mathrm{m}$ pixel size). The spot size has been measured to be almost the same for all the gratings. (a) Spot image. (b) Cross sections along the spot profile.

levels, and the monochromator resolving power is high enough to fully resolve the $4 p_{3 / 2}(8.6 \mathrm{eV})$ and $4 p_{1 / 2}(9.3 \mathrm{eV})$ lines.

$\mathrm{Kr} 4 p$ photoelectron spectra as a function of the XUV-IR delay are shown in Fig. 11, as an example of experimental data obtained with the different gratings. The $4 p_{3 / 2}$ and $4 p_{1 / 2}$ peaks are related to single-photon photoionization and do not depend on the presence of the IR dressing pulse. When the IR-XUV pulses are temporally overlapped, sidebands appear at 7.1 and $10.1 \mathrm{eV}$ for the $4 p_{3 / 2}$ state and at 7.8 and $10.8 \mathrm{eV}$ for the $4 p_{1 / 2}$ state. The intensity of each sideband as a function of the delay is the convolution of the XUV and IR pulses, from which the duration of the XUV pulse may be calculated. The resulting XUV duration is reported in Table 3. Note that the duration measured here is the convolution between the pulse duration as entering into the DCM, the pulse front tilt given by the monochromator, and the wavefront distorsions as given by the
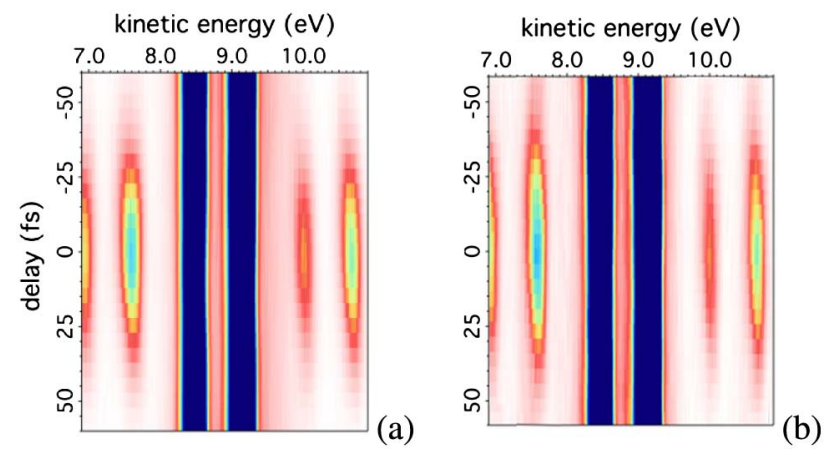

Fig. 11. $\mathrm{Kr} 4 p$ photoelectron spectra as a function of the XUV-IR delay, beam monochromatized at H15, $23.3 \mathrm{eV}$. (a) OPM G200 grating. (b) OPM G400 grating. 
Table 3. Pulse Duration of $\mathrm{H} 15(23.3 \mathrm{eV})$ at Output of DCM

\begin{tabular}{lccc}
\hline Grating & $\begin{array}{c}\text { Measured Pulse } \\
\text { Duration (fs) }\end{array}$ & $\begin{array}{c}\text { Expected Pulse } \\
\text { Front Tilt (fs) }\end{array}$ & $\begin{array}{c}\text { Bandwidth, } \\
100 \mu \mathrm{m} \text { Slit }(\mathrm{eV})\end{array}$ \\
\hline OPM, & $23 \pm 3$ & 18 & 0.55 \\
G200 & $35 \pm 3$ & 36 & 0.27 \\
OPM. & $35 \pm 55$ & 54 & 0.18 \\
G400 & $170 \pm 10$ & 150 & 0.07 \\
OPM, & & & \\
G600 & CDM, & & \\
G300 & & & \\
\hline
\end{tabular}

${ }^{a} \mathrm{IR}$ pulse duration has been assumed to be $40 \mathrm{fs}$. The bandwidth is calculated on a $100 \mu \mathrm{m}$ slit.

optical aberrations. The latter effect, as discussed in Section 4.C, may contribute to the output pulse duration of a few femtoseconds. Therefore, the main effect contributing to the broadening of the instrument temporal response is the pulse front tilt.

It has been verified that the duration is not influenced by the width of the slit for apertures in the range of 50-200 $\mu \mathrm{m}$, confirming that the main factor determining the duration is the pulse front tilt.

Since the beam divergence has been measured to be $1.3 \mathrm{mrad} \mathrm{FWHM}$ at $\mathrm{H} 15$, it is possible to calculate the expected pulse front tilt at the output. The calculated values are reported in Table 3 . In the case of the OPM gratings, the agreement between the expected front tilt and the measured pulse duration is good for OPM G200 and OPM G400. In the case of the OPM G600, the measured duration is lower than the expected pulse front tilt. This has been ascribed to a partial cut of the diffracted beam in the circular aperture placed after the grating to let propagate the 1st order diffracted beam and stop the IR beam in the 0 th order. In the case of CDM gratings, the measurement has been performed only with CDM G300, and the agreement between the measurement and the expected duration is indeed good.

Assuming at H23, the photon flux as reported in Section 4.B with the OPM G400 grating, i.e., $0.57 \mathrm{~nJ} /$ pulse, the spot size as reported in Section 4.C, i.e., $130 \times 220 \mu \mathrm{m}^{2}=2.86 \cdot 10^{-4} \mathrm{~cm}^{2}$, and a pulse duration of $25 \mathrm{fs}$, where the measured duration (35 fs at H15) has been linearly scaled with the wavelength, the maximum achievable peak intensity on the XUV focus results in $8 \cdot 10^{7} \mathrm{~W} / \mathrm{cm}^{2}$.

The measurements confirm the capability of the DCM to operate in two different regimes: ultrafast response and large bandwidth using the OPM stage or longer response and narrow bandwidth using the CDM stage.

\section{Conclusion}

The design and characterization of a double-configuration monochromator has been presented. Two grating geometries have been joined together in a single instrument to realize a monochromator, which is capable of giving ultrafast response and high pho- ton flux with low spectral resolution or high resolution with longer response and lower flux.

When compared with other schemes used to obtain a monochromatized XUV beam, such as the use of multilayer mirrors, the use of grating monochromators gives higher spectral purity and wide tunability over a broad range. The double-configuration monochromator gives additional flexibility in choosing energy resolution versus temporal resolution.

The authors thank Carla Puglia and Antti Kivimäki for assistance in setting up the photoemission experimental setup. The Department of Physics and Astronomy of Uppsala University is acknowledged for providing the Scienta SES-200 spectrometer in the framework of the collaboration with CNR-IOM (TASC laboratory, Gas Phase beamline, Trieste). The CITIUS project is funded by the program for cross-border cooperation between Italy and Slovenia 2007-2013, Strategic Projects.

\section{References}

1. P. Jaeglè, Coherent Sources of XUV Radiation (Springer, 2006).

2. Z. Chang, A. Rundquist, H. Wang, M. M. Murnane, and H. C. Kapteyn, "Generation of coherent soft $\mathrm{X}$ rays at $2.7 \mathrm{~nm}$ using high harmonics," Phys. Rev. Lett. 79, 2967-2970 (1997).

3. C. Spielmann, N. H. Burnett, S. Sartania, R. Koppitsch, M. Schnuerer, C. Kan, M. Lenzner, P. Wobrauschek, and F. Krausz, "Generation of coherent X-rays in the water-window using 5-femtosecond laser pulse," Science 278, 661-664 (1997).

4. G. Sansone, L. Poletto, and M. Nisoli, "High-energy attosecond light sources," Nat. Photonics 5, 655-663 (2011).

5. M. Chini, K. Zhao, and Z. Chang, "The generation, characterization and applications of broadband isolated attosecond pulses," Nat. Photonics 8, 178-186 (2014).

6. F. Krausz and M. I. Stockman, "Attosecond metrology: from electron capture to future signal processing," Nat. Photonics 8, 205-213 (2014).

7. T. Sekikawa, T. Okamoto, E. Haraguchi, M. Yamashita, and T. Nakajima, "Two-photon resonant excitation of a doubly excited state in He atoms by high-harmonic pulses," Opt. Express 16, 21922-21929 (2008).

8. P. Villoresi, "Compensation of optical path lengths in extremeultraviolet and soft-x-ray monochromators for ultrafast pulses," Appl. Opt. 38, 6040-6049 (1999).

9. L. Nugent-Glandorf, M. Scheer, D. A. Samuels, V. Bierbaum, and S. R. Leone, "A laser-based instrument for the study of ultrafast chemical dynamics by soft x-ray-probe photoelectron spectroscopy," Rev. Sci. Instrum. 73, 1875-1886 (2002).

10. J. Norin, K. Osvay, F. Albert, D. Descamps, J. Yang, A. L'Huillier, and C. Wahlstrom, "Design of an extreme-ultraviolet monochromator free from temporal stretching," Appl. Opt. 43, 1072-1081 (2004).

11. L. Poletto, "Time-compensated grazing-incidence monochromator for extreme-ultraviolet and soft x-ray high-order harmonics," Appl. Phys. B 78, 1013-1016 (2004).

12. L. Poletto and P. Villoresi, "Time-compensated monochromator in the off-plane mount for extreme-ultraviolet ultrashort pulses," Appl. Opt. 45, 8577-8585 (2006).

13. L. Poletto, P. Villoresi, E. Benedetti, F. Ferrari, S. Stagira, G. Sansone, and M. Nisoli, "Intense femtosecond extreme ultraviolet pulses by using a time-delay compensated monochromator," Opt. Lett. 32, 2897-2899 (2007).

14. L. Poletto, P. Villoresi, F. Frassetto, F. Calegari, F. Ferrari, M. Lucchini, G. Sansone, and M. Nisoli, "Time-delay compensated monochromator for the spectral selection of extremeultraviolet high-order laser harmonics," Rev. Sci. Instrum. 80, 123109 (2009). 
15. M. Ito, Y. Kataoka, T. Okamoto, M. Yamashita, and T. Sekikawa, "Spatiotemporal characterization of single-order high harmonic pulses from time-compensated toroidalgrating monochromator," Opt. Express 18, 6071-6078 (2010).

16. H. Igarashi, A. Makida, M. Ito, and T. Sekikawa, "Pulse compression of phase-matched high harmonic pulses from a time-delay compensated monochromator," Opt. Express 20, 3725-3732 (2012).

17. L. Poletto and F. Frassetto, "Time-preserving monochromators for ultrafast extreme-ultraviolet pulses," Appl. Opt. 49, 5465-5473 (2010).

18. C. Grazioli, C. Callegari, A. Ciavardini, M. Coreno, F. Frassetto, D. Gauthier, D. Golob, R. Ivanov, A. Kivimäki, B. Mahieu, B. Bučar, M. Merhar, P. Miotti, L. Poletto, E. Polo, B. Ressel, C. Spezzani, and G. De Ninno, "CITIUS: an infrared-extreme ultraviolet light source for fundamental and applied ultrafast science," Rev. Sci. Instrum. 85, 023104 (2014).

19. W. Werner, "X-ray efficiencies of blazed gratings in extreme off-plane mountings," Appl. Opt. 16, 2078-2080 (1977).

20. M. Neviere, D. Maystre, and W. R. Hunter, "On the use of classical and conical diffraction mountings for XUV gratings," J. Opt. Soc. Am. 68, 1106-1113 (1978).

21. W. Cash and R. Kohnert, "Very high X-ray efficiency from a blazed grating," Appl. Opt. 21, 17-18 (1982).

22. J. F. Seely, L. I. Goray, B. Kjornrattanawanich, J. M. Laming, G. E. Holland, K. A. Flanagan, R. K. Heilmann, C.-H. Chang, M. L. Schattenburg, and A. P. Rasmussen, "Efficiency of a grazing-incidence off-plane grating in the soft-x-ray region," Appl. Opt. 45, 1680-1687 (2006).
23. L. Poletto and F. Frassetto, "Design of high-resolution grazing-incidence echelle monochromators," Appl. Opt. 48, 5363-5370 (2009).

24. E. G. Loewen and E. Popov, Diffraction Gratings and Applications (Marcel Dekker, 1997).

25. M. Pascolini, S. Bonora, A. Giglia, N. Mahne, S. Nannarone, and L. Poletto, "Gratings in the conical diffraction mounting for an EUV time-delay compensated monochromator," Appl. Opt. 45, 3253-3562 (2006).

26. R. Haight and D. R. Peale, "Tunable photoemission with harmonics of subpicosecond lasers," Rev. Sci. Instrum. 65, 1853-1857 (1994).

27. F. Frassetto, C. Cacho, C. A. Froud, I. C. E. Turcu, P. Villoresi, W. A. Bryan, E. Springate, and L. Poletto, "Grating monochromator for extreme-ultraviolet ultrashort pulses," Opt. Express 19, 19169-19181 (2011).

28. P. Salières, T. Ditmire, M. D. Perry, A. L'Huillier, and M. Lewenstein, "Angular distributions of high-order harmonics generated by a femtosecond laser," J. Phys. B 29, 4771 (1996).

29. T. E. Glover, R. W. Schoenlein, A. H. Chin, and C. V. Shank, "Observation of laser assisted photoelectric effect and femtosecond high order harmonic radiation," Phys. Rev. Lett. 76, 2468-2471 (1996).

30. E. S. Toma, H. G. Muller, P. M. Paul, P. Breger, M. Cheret, P. Agostini, C. Le Blanc, G. Mullot, and G. Cheriaux, "Ponderomotive streaking of the ionization potential as a method for measuring pulse durations in the XUV domain with fs resolution," Phys. Rev. A 62, 061801 (2000). 